\title{
Salmonella enterica subspecies enterica serovar Enteritidis Salmonella pathogenicity island 2 type III secretion system: role in intestinal colonization of chickens and systemic spread
}

Correspondence

Wolfgang Köster

wolfgang.koester@usask.ca

Received 8 January 2010

Revised 11 May 2010

Accepted 18 May 2010

\author{
Amanda L. S. Wisner, Taseen S. Desin, Birgit Koch, ${ }^{\dagger}$ Po-King S. Lam, \\ Emil M. Berberov, Claudia S. Mickael, Andrew A. Potter \\ and Wolfgang Köster
}

Vaccine and Infectious Disease Organization, University of Saskatchewan, 120 Veterinary Road, Saskatoon, SK, Canada S7N 5E3

\begin{abstract}
Salmonella enterica subspecies enterica serovar Enteritidis ( $S$. Enteritidis) has been identified as a significant cause of salmonellosis in humans. Salmonella pathogenicity islands 1 and 2 (SPI-1 and SPI-2) each encode a specialized type III secretion system (T3SS) that enables Salmonella to manipulate host cells at various stages of the invasion/infection process. For the purposes of our studies we used a chicken isolate of $S$. Enteritidis (Sal18). In one study, we orally co-challenged 35-day-old specific pathogen-free (SPF) chickens with two bacterial strains per group. The control group received two versions of the wild-type strain Sal18: Sal18 attTn7:: tet and Sal18 attTn7:: cat, while the other two groups received the wild-type strain (Sal18 attTn $7::$ tet) and one of two mutant strains. From this study, we concluded that $S$. Enteritidis strains deficient in the SPI1 and SPI-2 systems were outcompeted by the wild-type strain. In a second study, groups of SPF chickens were challenged at 1 week of age with four different strains: the wild-type strain, and three other strains lacking either one or both of the SPI-1 and SPI-2 regions. On days 1 and 2 post-challenge, we observed a reduced systemic spread of the SPI-2 mutants, but by day 3 , the systemic distribution levels of the mutants matched that of the wild-type strain. Based on these two studies, we conclude that the S. Enteritidis SPI-2 T3SS facilitates invasion and systemic spread in chickens, although alternative mechanisms for these processes appear to exist.
\end{abstract}

\section{INTRODUCTION}

Salmonella enterica subspecies enterica serovar Enteritidis (also known as $S$. Enteritidis) is an important human pathogen that causes salmonellosis in humans. S. Enteritidis is passed to humans mainly via poultry meat contaminated at the time of slaughter, and also via consumption of contaminated water. In addition, eggs are often heavily colonized by $S$. Enteritidis, and humans can contract salmonellosis via consumption of raw or partially cooked eggs. Chickens colonized with $S$. Enteritidis typically do not show disease symptoms, and whole flocks can become infected quickly, shedding bacteria in their faeces for months (Catarame et al., 2005; Clavijo et al., 2006).

tPresent address: Department of Science, Systems and Models, Roskilde University, Building 27, Universitetsvej 1, DK-4000 Roskilde, Denmark.

Abbreviations: SCV, Salmonella-containing vesicle; SPF, specific pathogen-free; SPI, Salmonella pathogenicity island; T3SS, type III secretion system.
Infection caused by $S$. enterica is the second most common cause of bacterial gastroenteritis (food poisoning) in the developed world, and results in significant economic loss to the poultry industry, as well as placing a substantial burden on the healthcare system (Catarame et al., 2005; Meenakshi et al., 1999). Because there is a need to control the spread of $S$. Enteritidis, the aim of our project was to focus our efforts at the beginning of the chain of infection: colonization of the chicken. It has been estimated that there are approximately 1.4 million cases of salmonellosis per year in the USA, resulting in approximately 15000 hospitalizations and 400 deaths per year (http://www.ers. usda.gov/data/foodborneillness/salm_Intro.asp). An estimated $95 \%$ of the cases are contracted from contaminated food or food products (Callaway et al., 2008). S. Enteritidis is responsible for $15 \%$ of the cases, and is the second most common serovar isolated from poultry in North America, and the most common serovar isolated from humans in the European Union (Foley et al., 2008; Vieira, 2009). As S. Enteritidis is passed to humans mainly through consumption of contaminated poultry meat and eggs, it is important 
to better understand the pathogenesis of $S$. Enteritidis in chickens in order to prevent it.

S. Enteritidis is known to use two specialized type III secretion systems (T3SSs) that are thought to facilitate invasion and survival within the host cell. The two T3SSs are encoded within Salmonella pathogenicity islands 1 and 2 (SPI-1 and SPI-2). The T3SSs secrete effectors into the host cell, triggering a number of events in the infected cell that culminate in the symptoms of enteritis in humans: fever, diarrhoea and abdominal pain. It is the current view that the SPI-1 T3SS is mainly involved in invasion of the host cell, while the SPI-2 T3SS plays a role in survival within the host cell, and maintenance of the Salmonellacontaining vesicle (SCV) (Brown et al., 2005; Chakravortty et al., 2005).

SPI-2 is a region of approximately $40 \mathrm{~kb}$ located at centisome 31 in Salmonella species, and has been reported to be necessary for systemic infection (Galán, 2001). The SPI-2 region encodes 44 proteins that are essential for intracellular proliferation, survival, and maintenance of the SCV, as well as its own regulatory system, SsrA/B (Olekhnovich \& Kadner, 2006; Thompson et al., 2006; Winstanley \& Hart, 2001). SPI-2 effectors are secreted across the SCV membrane and stop fusion of lysosomes with the SCV, thereby avoiding reactive oxygen speciesand NADPH oxidase-mediated killing of the bacteria (Babu et al., 2006; Coombes \& Finlay, 2005; Kuhle \& Hensel, 2004). Effectors facilitate systemic spread and the maturation of the SCV, and can act as pro- or antiinflammatory factors (McGhie et al., 2009; Schlumberger \& Hardt, 2006). Once in the mature SCV, Salmonella proliferates, and it can traverse the epithelial cell and invade underlying tissue after being released on the basolateral side (Fortune et al., 2006; Guiney, 2005).

Mutations that disrupt the SPI-2 T3SS have been shown to result in highly attenuated virulence, as well as defective growth in macrophages and epithelial cells (Galán, 2001; Kuhle \& Hensel, 2004; Shah et al., 2005). SPI-2 T3SS expression in culture is optimal in acidic, low osmolarity, low $\mathrm{Ca}^{2+}$, minimal nutrient conditions. These conditions mimic the SCV environment (Chakravortty et al., 2005; Coombes et al., 2004; Galán, 2001). Unlike in the SPI-1 T3SS, the SPI-2 needle is extended by a filament composed of SseB subunits and is required for secretion of the translocation proteins ( $\mathrm{SseC}$ and $\mathrm{SseD}$ ) (Chakravortty et al., 2005; Coombes et al., 2005; Ghosh, 2004; Kuhle \& Hensel, 2004; Waterman \& Holden, 2003). At least 19 effectors are secreted by the SPI-2 T3SS, and most are encoded by genes located outside the SPI-2 locus (Kuhle \& Hensel, 2004; McGhie et al., 2009; Waterman \& Holden, 2003).

Until recently, it was widely accepted that of the two T3SSs, the SPI-1 T3SS was alone in its expression in the intestinal lumen, and SPI-2 T3SS expression was only induced after entry of Salmonella into the host cells and establishment of the SCV. However, a recent study in mice has shown that the SPI-2 T3SS is expressed in the intestinal lumen prior to attachment and entry of the bacteria, albeit to a much lower extent than that of SPI-1. This is probably an attempt by the bacterium to prepare its invasion arsenal (Brown et al., 2005). There have also been studies showing that the SPI-1 T3SS continues to be expressed once Salmonella is inside the SCV, and that some of the SPI-1 effector proteins work in conjunction with SPI-2 effector proteins to manipulate the host cell (Brawn et al., 2007; Giacomodonato et al., 2007; Hautefort et al., 2008). There have been many studies based on the reduction of colonization and shedding of Salmonella in chickens; however, the majority of these studies have involved Salmonella enterica subspecies enterica serovars Typhimurium, Pullorum and Gallinarum (Jones et al., 2001, 2007; Lee et al., 2005; Rana \& Kulshreshtha, 2006; Turner et al., 1998; Wigley et al., 2002).

The reduced ability of the SPI-2 knockout mutants produced in our laboratory to colonize chickens has provided us with knowledge about the importance of the SPI-2 T3SS in the colonization process. Using these mutants, we have observed that in a co-challenge situation, the wild-type Salmonella strain outcompetes the mutant for systemic spread, as measured by recovering the bacteria from liver and spleen. Using a single-challenge model, we have observed that although the SPI-2 T3SS plays an important role in systemic infection, it is not the only factor involved in this process.

\section{METHODS}

Bacterial strains and plasmids. Bacterial strains and plasmids used in this study are described in Table 1 . Standard growth procedures were followed using Luria-Bertani (LB) broth and agar at $37^{\circ} \mathrm{C}$. Media were supplemented with antibiotics (Sigma) when required: ampicillin, 100 and $50 \mu \mathrm{g} \mathrm{ml}^{-1}$; tetracycline, 10 and $5 \mu \mathrm{g} \mathrm{ml}{ }^{-1}$; chloramphenicol, 30 and $9 \mu \mathrm{g} \mathrm{ml}^{-1}$; streptomycin, $25 \mu \mathrm{g} \mathrm{ml}^{-1}$.

Primers. All primers used in this study were synthesized by Invitrogen, and designed based on the $S$. Enteritidis PT4 sequence provided by the Wellcome Trust Sanger Institute (GenBank accession number AM933172) (Thomson et al., 2008). Table 2 provides a list of primers used in this study.

PCR. All PCRs were carried out using Taq polymerase (New England Biolabs), applying reaction conditions suggested by the supplier.

Cloning. Flanking regions of spaS and ssaU were amplified using the following primers: spaS flank 1 (OL 1-23 and OL 1-25), spaS flank 2 (OL 1-22 and OL 1-24), ssaU flank 1 (OL 1-19 and OL 1-21), and ssaU flank 2 (OL 1-18 and OL 1-26). During PCR, restriction sites were added to these flanking regions, resulting in the products HindIII-Flank 1-XbaI and XbaI-Flank 2-EcoRI. These products were inserted into the pGEM-T vector (Promega) for confirmation of the correct sequence (data not shown). The resulting plasmids (one harbouring the left-flanking region of the gene of interest, the other the right-flanking region) were cut with the restriction enzymes $\mathrm{XbaI}$ and ScaI. The fragments of the plasmids containing the flanking regions were then ligated together. This created a plasmid containing both the right- and left-flanking regions of the gene of interest directly. All regions of the pGEM-T vector remained intact, most 
Table 1. Bacterial strains and plasmids

\begin{tabular}{|c|c|c|}
\hline Strain or plasmid & Description & Source \\
\hline \multicolumn{3}{|l|}{ Strains } \\
\hline E. coli $\mathrm{DH} 5 \alpha$ & $\begin{array}{l}\mathrm{F}^{-} \phi 80 \mathrm{~d} l a c Z \Delta \mathrm{M} 15 \Delta(\text { lacZYA-argF }) \mathrm{U} 169 \text { deoR recA1 endA1 hsdR17 }\left(\mathrm{r}_{\mathrm{k}}^{-} \mathrm{m}_{\mathrm{k}}^{-}\right) \\
\text {phoA supE44 } \lambda^{-} \text {thi-1 gyrA96 relA1 }\end{array}$ & Invitrogen \\
\hline E. coli $\mathrm{BL}-21(\mathrm{DE} 3)$ & $\mathrm{F}^{-}$ompT hsdSB $\left(\mathrm{r}_{\mathrm{B}}^{-} \mathrm{m}_{\mathrm{B}}^{-}\right) g a l d c m(\mathrm{DE} 3)$ & Invitrogen \\
\hline E. coli BL-21(pLysS) & $\mathrm{F}^{-} \operatorname{ompT} h s d S B\left(\mathrm{r}_{\mathrm{B}}^{-} \mathrm{m}_{\mathrm{B}}^{-}\right) g a l d c m(\mathrm{DE} 3)$ pLysS (CamR) & Invitrogen \\
\hline S. Enteritidis Sal18 & Virulent for birds; invades spleen and liver and colonizes gut & C. Poppe $e^{*}$ \\
\hline S. Typhimurium SL1344 & Wild-type, streptomycin-resistant & B. Finlay $\dagger$ \\
\hline Sal18 att $\operatorname{Tn} 7::$ tet & Sal18 with tetracycline-resistance cassette inserted at $g \operatorname{lm} S$ site & This study \\
\hline Sal18 $\operatorname{att} \operatorname{Tn} 7:: c a t$ & Sal18 with chloramphenicol-resistance gene inserted at $g \operatorname{lmS}$ site & This study \\
\hline Sal18 att $\operatorname{Tn} 7:$ : cat $\Delta s p a S \Delta s s a U$ & $\begin{array}{l}\text { Sal18 with deletion of spaS and ssaU genes, and chloramphenicol-resistance } \\
\text { gene inserted at glms site }\end{array}$ & This study \\
\hline Sal18 $\Delta$ SPI-2: : cat & $\begin{array}{l}\text { Sal18 with whole SPI- } 2 \text { region replaced by a chloramphenicol-resistance gene } \\
\text { by using the } \lambda \text { Red system }\end{array}$ & This study \\
\hline pGEM-T & $\begin{array}{l}\text { Has single } 3^{\prime}-\mathrm{T} \text { overhangs at the insertion site. These greatly improve the } \\
\text { efficiency of ligation of a PCR product into the plasmid by preventing } \\
\text { recircularization of the vector and providing a compatible overhang for } \\
\text { ligation of PCR products generated by certain thermostable polymerases. } \\
\text { Can be used for Blue/White screening (Promega) }\end{array}$ & Promega \\
\hline pHSG415 & Temperature-sensitive & $\begin{array}{l}\text { A. P. White and M. } \\
\text { G. Surette } \ddagger\end{array}$ \\
\hline pET-15b & His-tag vector, contains ampicillin-resistance cassette & Novagen \\
\hline pBR322 & Cloning vector, contains ampicillin- and tetracycline-resistance cassettes & New England Biolabs \\
\hline pKD3 & Used in $\lambda$ Red system & $\begin{array}{l}\text { A. P. White and M. } \\
\text { G. Surette }\end{array}$ \\
\hline
\end{tabular}

${ }^{\star}$ Laboratory for Foodborne Zoonoses, Health Canada, Guelph, ON, Canada.

$\dagger$ University of British Columbia, Vancouver, BC, Canada.

$\ddagger$ University of Calgary, Calgary, $\mathrm{AB}$, Canada.

importantly the ampicillin-resistance gene and the origin of replication. The combined right- and left-flanking regions were excised from the pGEM-T vector and placed into the temperaturesensitive plasmid pHSG415 by using the HindIII and EcoRI restriction sites. The resulting plasmid was electroporated into wild-type Sal18 competent cells using standard techniques (Electro Cell Manipulator 630, BTX Harvard Apparatus). Recombination of the pHSG415 plasmid harbouring the flanking regions of the genes of interest was induced (Fig. 1) as described elsewhere (White et al., 2007), and correct recombinants were selected.

The left- and right-flanking regions of the SPI-2 region were assembled with the tetracycline-resistance gene from plasmid pBR322 into the pGEM-T vector, as described above. The resulting plasmid contained the amplified products in the following order: SPI2 left flank, tetracycline-resistance cassette, SPI-2 right flank. The tetracycline-resistance cassette, including the flanking regions, was then amplified via PCR (by using primers OL 2-69 and OL 2-70), and the resulting PCR product of $4286 \mathrm{bp}$ was used in the $\lambda$ Red system (Datsenko \& Wanner, 2000).

For protein expression purposes, the sseB gene was amplified by PCR (using primers OL 1-35, OL 1-36, OL 1-37 and OL 1-38), and cloned into the pGEM-T vector for sequencing. Once the presence of the genes had been confirmed by sequencing and restriction gel analysis (data not shown), the genes were excised, and inserted into the pET$15 \mathrm{~b}$ vector (Novagen) by using the $\mathrm{Bam} \mathrm{HI}$ and $\mathrm{Ncol}$ restriction sites.

Construction of mutants using the $\lambda$ Red system. The $\lambda$ Red system is an efficient and widely used method for the inactivation of chromosomal genes in Escherichia coli and in S. Typhimurium (Datsenko \& Wanner, 2000; Hansen-Wester et al., 2004; Murphy \& Campellone, 2003; Tischer et al., 2006). This system was used to produce two SPI-2 knockout mutants. The SPI-2 knockout mutant Sal18 $\Delta$ SPI-2 : : cat was produced using primers OL 2-69 and OL 2-70, which were designed based on the protocol originally described by Datsenko \& Wanner (2000). A SPI-1 knockout mutant, Sal18 $\Delta$ SPI$1:: c a t$, and a SPI-1/SPI-2 knockout mutant, Sal18 $\Delta$ SPI-1 $\Delta$ SPI- $2:: c a t$, were produced using the same method. A chloramphenicol-resistance marker was also added to Sal18 (Sal18 attTn7::cat) and the $\Delta s p a S \Delta s s a U$ strain (Sal18 att $\operatorname{Tn} 7::$ cat $\Delta s p a S \Delta s s a U$ ) by inserting the chloramphenicol-resistance gene into an intergenic region downstream of the glucosamine-6-phosphate synthase gene $(\mathrm{glmS})$ using the $\lambda$ Red method. This site was chosen because it represents the Tn 7 insertion site, and insertions at this position are unlikely to interfere with cellular 
Table 2. Primers

The table shows all primers used in this study for amplification of certain areas in the SPI-2 region of the S. Enteritidis 18 chromosome and for sequencing. Recognition sequences for restriction enzymes are in bold type.

\begin{tabular}{|c|c|c|c|}
\hline Primer no. & Primer name & Primer sequence $\left(5^{\prime} \rightarrow 3^{\prime}\right)$ & $\begin{array}{c}\text { Restriction } \\
\text { enzyme }\end{array}$ \\
\hline OL $1-35$ & sseBCD Fwd & AACCGCAGCGTACACGTAGT & None \\
\hline OL $1-37$ & sseB Fwd & CATATGTCTTCAGGAAACATCTT & NdeI \\
\hline OL $1-38$ & sseB Bwd & GGATCCTCATGAGTACGTTTTC & BamHI \\
\hline OL $1-44$ & SPI2 Left Flank Fwd & AAGCTTGGACATGGCTGCCGTCGCTATC & HindIII \\
\hline OL 2-63 & SPI2 Right Flank Fwd & AGATCTGCGACGGCGATTTC & $B g l I I$ \\
\hline OL 2-64 & SPI2 Right Flank Bwd & GAATTCCGCGCATTATACGCTG & EcoRI \\
\hline OL $1-48$ & pBR322 Tet $^{\mathrm{R}}$ Fwd & AGATCTGTTTGACAGCTTATCATCGATAGGC & $B g l I I$ \\
\hline OL $1-49$ & pBR322 Tet $^{R}$ Bwd & GGTACCAATTCTTGGAGTGGTGAATC & $K p n \mathrm{I}$ \\
\hline OL $1-23$ & spaS Flank 1-1 & GCTCTAGAGGCAGTAGCGATGTATTC & $X b a \mathrm{I}$ \\
\hline OL $1-25$ & spaS Flank 1-2 & TATAAGCTTGCCTCAGCGAGGCGCGG & HindIII \\
\hline OL $1-18$ & ssaU Flank 2-1 & TCTCTAGACAGATGGAAACCAGTC & $X b a \mathrm{I}$ \\
\hline OL $1-26$ & ssaU Flank 2-2 & TCGAATTCAGCAGCAACAGG & EcoRI \\
\hline OL 3-25 & $\lambda$ Red SPI1 Fwd & $\begin{array}{l}\text { GCTGTCGCGTATGAAGCGATTGGGTATTGATAAAG- } \\
\text { ACGCGTTAGCGTAAGTGTAGGCTGGAGCTGCTTC }\end{array}$ & None \\
\hline OL 3-26 & $\lambda$ Red SPI1 Bwd & $\begin{array}{l}\text { ATATGGTCTTAATTATATCATGATGAGTTCAGCCAA- } \\
\text { CGGTGATATGGCCCATATGAATATCCTCCTTA }\end{array}$ & None \\
\hline OL 2-69 & $\lambda$ Red SPI2 Fwd & $\begin{array}{l}\text { TCCAGGACGCGTGGTATTGGCATATCGGTGGGATGA- } \\
\text { TAGCCAAGACAAACGTGTAGGCTGGAGCTGCTTC }\end{array}$ & None \\
\hline OL 2-70 & $\lambda$ Red SPI2 Bwd & $\begin{array}{l}\text { TGCCTCGCTCTAAGGATAGGTGACATCGAAAGAGCGT- } \\
\text { GCAGAGGAATGTGCATATGAATATCCACCTTAG }\end{array}$ & None \\
\hline OL 3-38 & $g \operatorname{lm} S A B C$ Fwd & $\begin{array}{l}\text { AGCGCAGGTAGGCGTAGCACCTCTTAGTCGCTCTTCA- } \\
\text { GCCACCATAGAGAGTGTAGGCTGGAGCTGCTTC }\end{array}$ & None \\
\hline
\end{tabular}

functions (Craig, 1991). Briefly, PCR primers (OL 3-38 and OL 3-39) were designed that recognized the intergenic region downstream of the glmS gene, based on the S. Enteritidis PT4 NCTC 13349 sequence provided by the Wellcome Trust Sanger Institute, and the chloramphenicol-resistance gene (pKD3) (Datsenko \& Wanner, 2000). A tetracycline-resistance gene from pBR322 was also added to Sal18 (Sal18 att $\operatorname{Tn} 7:$ : tet) by using the method described above.

Strain and construct confirmation. DNA sequencing was carried out on all constructs and PCR products using standard techniques (Desin et al., 2009).

Animal treatment. All protocols involving animals were carried out according to the guidelines provided by the University Council on Animal Care, protocol number 1994-213.

Protein and antibody production. The SseB protein was produced and purified as an N-terminal His-tag fusion protein by using standard protocols, as described previously by our group (Desin et al., 2009).

Induction of the SPI-2 T3SS and Western blotting. Strains were subjected to SPI-2-inducing conditions, as described, with minor modifications (Coombes et al., 2004). The cell lysate (pellet), total membrane and culture supernatant (secreted) fractions were isolated using standard techniques, separated on a SDS-polyacrylamide gel, and visualized by staining with Coomassie Brilliant Blue G-250 (BioRad). Proteins of the pellet and secreted fractions were transferred to nitrocellulose membranes (Bio-Rad) using a Semi-Dry Transfer Cell (Bio-Rad) according to directions provided by the supplier. Western blot analysis was carried out as suggested for the Odyssey Infrared Imaging System (LI-COR Biosciences). The primary antibodies used were polyclonal anti-SseB raised in rabbits and monoclonal mouse anti-DnaK supplied by Kirkegaard and Perry Laboratories. The secondary antibodies used were IRDye $680 \mathrm{CW}$-conjugated goat polyclonal anti-rabbit IgG and IRDye 680CW-conjugated goat polyclonal anti-mouse IgG 800, supplied by LI-COR Biosciences. Once dry, membranes were scanned with the Odyssey Infrared Imaging System.

Passaging Salmonella strains. Strains of interest were streaked for single colonies and grown overnight in LB broth (supplemented with antibiotics) to $\mathrm{OD}_{600} 0.7$. The bacterial cell culture was centrifuged at $3500 \mathrm{~g}$ for $10 \mathrm{~min}$, and the cell pellet was resuspended in saline for a final count of $2 \times 10^{10}$ c.f.u. $\mathrm{ml}^{-1}$ in $1 \mathrm{ml}$ total volume. Chickens (two birds per strain) were orally challenged with $0.5 \mathrm{ml}$ of the above suspension. On days 2 and 4 after challenge, birds were euthanized and 


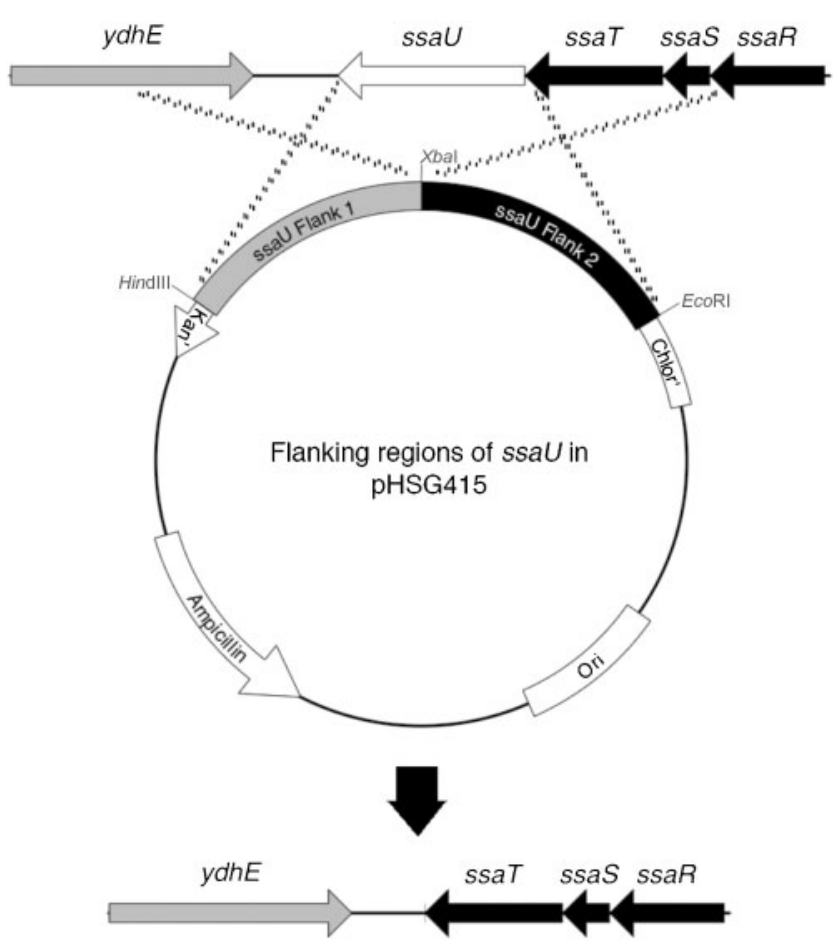

Fig. 1. Schematic representation of the pHSG415 method for gene replacement. Flanking regions of genes of interest were amplified using primers. The resulting products were inserted into the pGEM-T vector (Promega) for confirmation of the correct sequence (data not shown). The plasmids harbouring both the right- and left-flanking regions were cut with Xbal and Scal, and then ligated together. This created a plasmid containing both the right- and left-flanking regions of the gene of interest together. The combined right- and left-flanking regions were excised from the pGEM-T vector and inserted into the temperature-sensitive plasmid pHSG 415 by using the HindIII and EcoRI restriction sites.

tested for the presence of the bacterial strain of interest by swabbing the inside of sampled organs (liver, spleen and caecum), and plating on Brilliant Green agar. After incubation of the agar plates for $24 \mathrm{~h}$, resulting Salmonella colonies were tested by colony PCR to confirm the identity of the challenge strain. Strains were suspended in $50 \%$ glycerol $(\mathrm{v} / \mathrm{v})$ and stored at $-80{ }^{\circ} \mathrm{C}$ for use in the following trials.

Recovery of Salmonella on Brilliant Green agar. All strains used in the trials described below were first subjected to plating on Brilliant Green agar, as well as LB agar with and without antibiotics, to determine plating efficiency and strain fitness. Recovery of Salmonella on Brilliant Green agar by direct plating was approximately $40 \%$ of recovery on LB agar. As the addition of antibiotics to Brilliant Green agar further reduced the recovery rate by an additional $10-20 \%$, it was decided not to use antibiotics with Brilliant Green agar for recovery of Salmonella from chickens in the trials below. Addition of antibiotics to LB agar did not affect recovery. Further, there was no difference in the recovery and plating efficiency of any of the strains used on either Brilliant Green or LB agar, or in their growth kinetics in LB broth (data not shown).

Co-infection trial. Specific pathogen-free (SPF) chickens were obtained from Charles River Laboratories, and placed in isolation rooms at the Vaccine and Infectious Disease Organization (VIDO), University of Saskatchewan. At 35 days of age, birds were separated into three groups of 21 birds each, and co-challenged orally with two strains, each at a concentration of $1 \times 10^{10}$ bacteria in $0.5 \mathrm{ml}$. Results obtained from previous trials with SPF chickens were used to determine the appropriate dose (data not shown). Birds received Sal18 att $\operatorname{Tn} 7::$ tet and Sal18 att $\operatorname{Tn} 7::$ cat, Sal18 att $\operatorname{Tn} 7::$ tet and Sal18 att $\operatorname{Tn} 7::$ cat $\Delta$ spaS $\Delta s s a U$, or Sal18 att $\operatorname{Tn} 7::$ tet and Sal18 $\Delta$ SPI-1 $\Delta$ SPI$2::$ cat. The challenge inoculum was subjected to plating on Brilliant Green agar, with subsequent re-streaking of colonies onto LB agar containing tetracycline, and LB agar containing chloramphenicol, in order to confirm that the challenge was, in fact, a $50 \% / 50 \%$ (c.f.u./ c.f.u.) mixture of the two strains in question (data not shown). Birds were euthanized on days 1, 2 and 4 after challenge, and their livers, spleens and caeca were tested for the presence of the strains of interest. Samples of liver and spleen were weighed and homogenized in $10 \mathrm{ml}$ PBS. Homogenized liver and spleen (100 $\mu \mathrm{l}$ volumes) were plated on Brilliant Green agar, and c.f.u. $g^{-1}$ values were calculated for each strain. Caecal contents were weighed, and vortexed in $5 \mathrm{ml}$ PBS, and $25 \mu \mathrm{l}$ volumes of $10^{0}, 10^{-1}, 10^{-2}, 10^{-3}, 10^{-4}$ and $10^{-5}$ serial dilutions were plated on Brilliant Green agar. Colonies recovered by direct plating on Brilliant Green agar were re-streaked onto LB containing tetracycline, and LB containing chloramphenicol, in order to differentiate the strains recovered. c.f.u. $\mathrm{g}^{-1}$ values were calculated for each strain. In addition, because the recovery of Salmonella on Brilliant Green agar was only $40 \%$, samples from the liver, spleen and caecum of each bird were enriched in selenite broth and incubated at $37{ }^{\circ} \mathrm{C}$ overnight. The next day, a loop of each culture was plated on Brilliant Green agar. These data were used to determine the total number of samples and birds positive for Salmonella.

Single infection trial. SPF chicken eggs were obtained from Charles River Laboratories, and were incubated at the Department of Poultry Science (University of Saskatchewan) for 21 days until hatching. On the day of hatching, birds were transferred to isolation rooms at VIDO. One week after transfer, 20 birds were euthanized, and their caeca were collected and tested for the presence of Salmonella by plating on Brilliant Green agar. At this time, five swabs per room were taken, again to test for the presence of contaminating Salmonella, as identified by plating on Brilliant Green agar. Groups of 40 birds were challenged orally at 7 days of age (day 0$)$ with one of the following strains: Sal18 att $\operatorname{Tn} 7::$ tet, Sal18 $\Delta$ SPI-1::cat, Sal18 $\Delta$ SPI-2::cat or Sal18 $\Delta$ SPI-1 $\Delta$ SPI-2: : cat, in $0.5 \mathrm{ml}$ doses of $10^{10}$ c.f.u. $\mathrm{ml}^{-1}$. On days 1, 2, 3 and 4 after challenge, 10 birds per group were euthanized, and their livers, spleens and caeca were sampled and plated as described above. Samples from the liver, spleen and caecum of each bird were enriched in selenite broth, as described above. Results pertaining to the group that received the total SPI-1 mutant (Sal18 $\Delta$ SPI-1::cat) have been reported previously by our group (Desin et al., 2009).

\section{RESULTS}

\section{Detection of SPI-2 T3SS proteins by Western blotting}

Wild-type S. Enteritidis Sal18 and its derivatives (Sal18 att $\operatorname{Tn} 7:$ : cat and Sal18 att $\operatorname{Tn} 7::$ tet) were positive for SseB in both the pellet fraction and the total membrane fraction, as expected (Fig. 2, lanes 1-3). The S. Enteritidis strains lacking the entire SPI-2 region were found to be negative for production of SseB (Fig. 2, lanes 4, 5 and 7). These results, along with those of DNA sequencing of PCR products (as described in Methods), confirmed that Sal18 

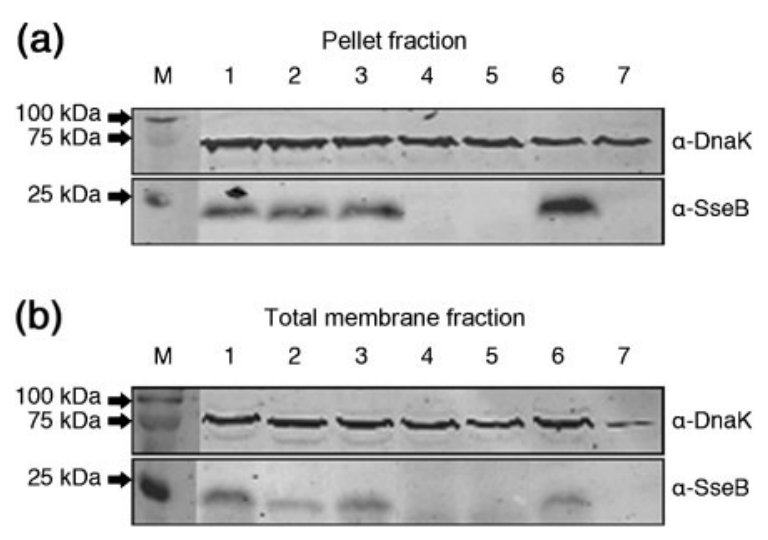

Fig. 2. Detection of SseB in pellet and total membrane fractions. The pellet and total membrane fractions of all strains shown in this figure were subjected to Western blot analysis. The membrane was first probed with rabbit polyclonal anti-SseB and monoclonal mouse anti-DnaK, and then with IRDye-labelled secondary antibodies, as described in Methods. (a) DnaK and SseB detected in the pellet fraction; (b) the same proteins detected in the total membrane fraction. Lanes: $M$, marker; 1, Sal18; 2, Sal18 attTn7::cat; 3, Sal18 attTn7::tet; 4, Sal18 $\Delta$ SPI-2; 5, Sal18 $\Delta$ SPI-2 : : cat; 6 , Sal18 attTn $7:$ : cat $\Delta s p a S \Delta s s a U ; 7$, Sal18 $\Delta$ SPI$1 \Delta \mathrm{SPI}-2:$ : cat.

$\Delta$ SPI-2:: cat and Sal18 $\Delta$ SPI-1 $\Delta$ SPI-2 : : cat were devoid of the SPI-2 region. The strain lacking only the ssaU gene within the SPI-2 region was found to be positive for production of SseB; SseB was found in both the pellet and the total membrane fractions of this strain (Fig. 2, lane 6). Since only the SsaU protein is missing in this strain, it is likely that the rest of the basal components (structural Ssa proteins and the ATPase SsaN) are assembled at the inner membrane. It is possible that SseB is still directed to the base of the incomplete SPI-2 T3SS by its chaperone, and is therefore associated with the total membrane fraction, as well as found free in the cytoplasm. Alternatively, SseB that is not secreted might form aggregates which co-purify with the total membrane fraction. SseB was not found in the secreted fraction of any strain. This is expected, as SseB is a part of the apparatus, and not a true secreted protein. It has been reported that strains lacking the $s s a U$ gene do not secrete SPI-2 effectors (Jones et al., 2001). DnaK was used as a loading control, and was found in the pellet fraction but not the secreted fraction, as expected. DnaK was also found in the total membrane fraction. DnaK is not normally associated with the membrane except under certain shock conditions (el Yaagoubi et al., 1994). In our study, the bacteria were under a certain amount of stress when they were switched from LB broth to SPI-2-inducing medium (low-phosphate medium), which had a low $\mathrm{pH}$ (5.6) and a different salt concentration compared with LB. Therefore, it is not surprising that DnaK was found associated with the total membrane fraction in this study.

\section{Co-challenge trial}

The co-challenge trial was carried out as described in Methods. Values of c.f.u. $\mathrm{g}^{-1}$ were calculated for Salmonella recovered from the liver, spleen and caecum of each bird (Fig. 3). Colonies were re-streaked on antibiotic-containing plates to determine the percentage of wild-type strain versus mutant strain in all organs sampled. On days 1, 3 and 4 post-challenge, there was no significant difference observed between the colonization levels of the two wild-type strains in the liver, spleen and caecum, as determined by a Wilcoxon signed rank test. This indicates that the antibiotic-resistance markers inserted into the wild-type genome (downstream of the $g \operatorname{lm} S$ gene) had no effect on the ability of the strains to colonize the caecum and spread systemically. On day 1 post-challenge, we saw no significant difference between the colonization levels of the wild-type and those of the mutant strain. On day 2 post-challenge, there was still no difference in caecal colonization. However, we found a significant difference $(P=0.01562)$ between the wild-type strain and the $\Delta$ spaS $\Delta s s a U$ strain concerning their recovery from both the liver and the spleen, with the wild-type strain outnumbering the mutant. Results of previous trials undertaken by our group have established that insertion of an antibiotic marker alone in the chromosome does not affect the performance of mutant strains compared with the wild-type (data not shown); therefore, the results seen in this trial were due to the introduced mutations only, and not to the introduction of different antibiotic markers. A significant difference was also observed $(P=0.03125)$ on day 2 post-challenge between the wild-type strain and the $\Delta$ SPI-1 $\Delta$ SPI-2 strain, again with the wild-type strain showing higher colonization levels. By day 4 , we saw a trend towards clearance of the mutant strain in the liver and the spleen, with $100 \%$ of the recovered colonies at this point belonging to the wild-type strain (Fig. 4). As discussed in Methods, strains were mixed equally before challenge. However, after enrichment, only one strain was found from spleens of the group that received strains Sal18 att $\operatorname{Tn} 7::$ tet and Sal18 att $\operatorname{Tn} 7::$ cat $\Delta s p a S \Delta s s a U$. This is likely a result of the poor recovery rate of Salmonella on Brilliant Green agar as well as of the small amount of sample used for enrichment ( $1 \mathrm{ml}$ out of $10 \mathrm{ml}$ total), because prior to enrichment, both strains were found in the spleen, as shown in Fig. 3(c).

\section{Single-challenge trial}

The single-challenge trial was carried out as described in Methods. Values of c.f.u. $\mathrm{g}^{-1}$ were determined for Salmonella isolated from the liver, spleen and caecum of all birds (Fig. 5). In addition, samples were enriched in selenite broth to reveal samples with numbers of Salmonella too low to be detected by direct plating (Fig. 6). A one-way ANOVA Kruskal-Wallis test was performed on the c.f.u. $\mathrm{g}^{-1}$ data for the liver, spleen and caecum. A $\chi^{2}$ Fisher's exact test was performed on the positive and 

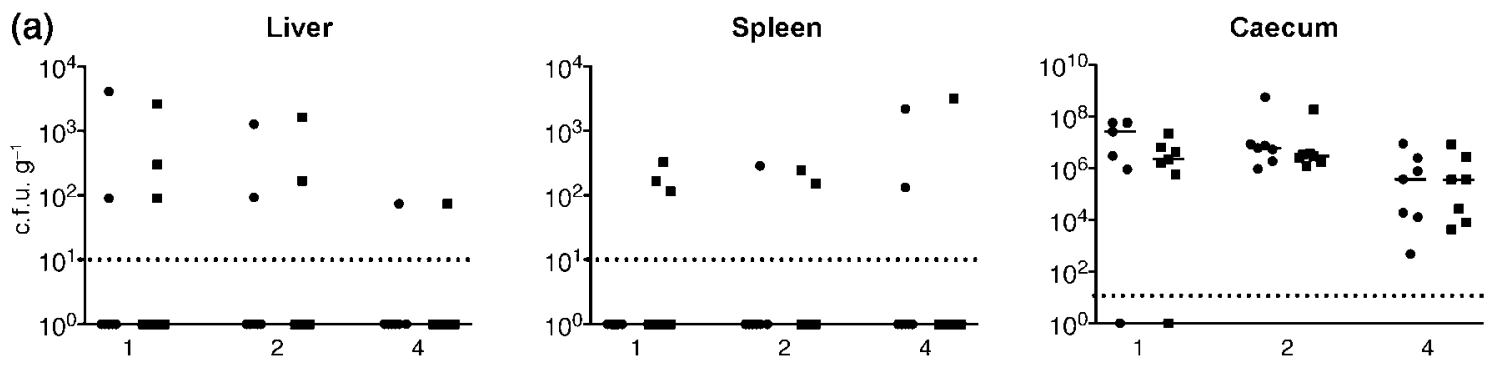

(b)
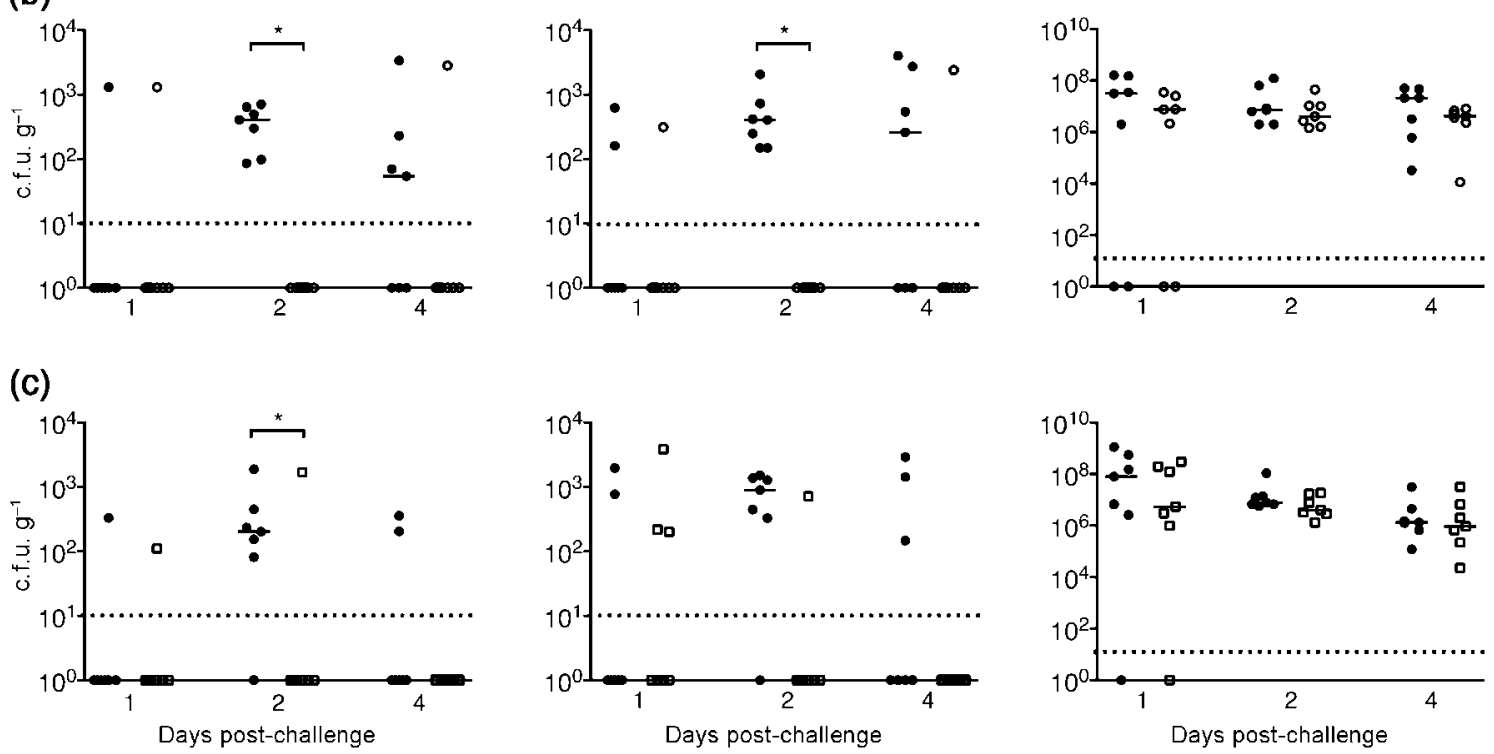

Fig. 3. Co-challenge trial: recovery of Salmonella from liver, spleen and caecum. (a) Calculated c.f.u. $\mathrm{g}^{-1}$ for recovered bacteria in the liver, spleen and caecum of birds challenged with wild-type Sal18 attTn7:: cat and wild-type Sal18 attTn $7::$ tet on days 1 , 2 and 4 post-challenge. (b) Calculated c.f.u. $\mathrm{g}^{-1}$ in the liver, spleen and caecum of birds challenged with wild-type Sal18 attTn $7:$ : tet and Sal18 attTn $7:$ : cat $\Delta s p a S \Delta s s a U$ on days 1, 2 and 4 post-challenge. (c) Calculated c.f.u. $\mathrm{g}^{-1}$ in the liver, spleen and caecum of birds challenged with wild-type Sal18 attTn $7:$ :tet and Sal18 $\Delta$ SPI-1 $\Delta$ SPI-2::cat on days 1, 2 and 4 postchallenge. We were unable to detect $S$. Enteritidis counts below $10^{1}$ c.f.u. $\mathrm{g}^{-1}$ by direct plating with our sampling method. This is shown by the dotted lines in the figure. Birds negative for countable c.f.u. after direct plating on Brilliant Green agar were assigned a value of 1 for graphical purposes. - Sal18 attTn7::tet; $\mathbf{0}$, Sal18 attTn7::cat; $\bigcirc$, Sal18 attTn7::cat $\Delta$ spaS $\Delta$ ssaU; $\square$, Sal18 $\Delta$ SPI-1 $\Delta$ SPI-2 : : cat. Asterisks indicate degree of statistical significance: ${ }^{*}, P<0.05 ;{ }^{\star *}, P<0.01$; $\star \star \star *, P<0.001$.

negative results. This was in order to determine significant differences in the colonization levels of each strain on days $1,2,3$ and 3. On day 1 post-challenge, no significant difference was observed between the c.f.u. $\mathrm{g}^{-1}$ of the wildtype strain and those of either of the mutant strains. However, there was a statistically significant difference in the number of birds that tested positive for Salmonella in the liver between the wild-type strain and the $\Delta$ SPI-2 strain $(P=0.0198)$, as well as between the wild-type strain and the $\Delta$ SPI-1 $\Delta$ SPI-2 strain $(P=0.0011)$. In both cases, the number of birds positive for Salmonella was higher in the group challenged with the wild-type strain than in the groups challenged with the mutant strains. On day 2 postchallenge, we saw a significant difference in the caecal c.f.u. $\mathrm{g}^{-1}$ between the group challenged with the wild-type strain and the group challenged with the $\Delta$ SPI-2 strain $(P=0.0111)$. Similar results were observed in the liver $(P=0.0001)$ and the spleen $(P=0.0108)$. The number of birds positive for Salmonella after enrichment coincided with these data, showing a higher number of birds with livers testing positive in the wild-type group versus the $\triangle$ SPI-2 group $(P=0.0001)$. The same was observed in the spleen $(P=0.0198)$. There was also a significant difference between the c.f.u. $\mathrm{g}^{-1}$ of the wild-type group and that of the $\Delta$ SPI-1 $\Delta$ SPI-2 group in both the liver $(P=0.0001)$ and the spleen $(P=0.0108)$. Again, enrichment data supported this observation, showing a higher number of birds with livers and spleens testing positive in the wild-type group than in the $\Delta$ SPI-1 $\Delta$ SPI-2 group $(P=0.0011$ and 0.0198 , respectively). Based on either c.f.u. $g^{-1}$ or enrichment data, 
(a)

Liver

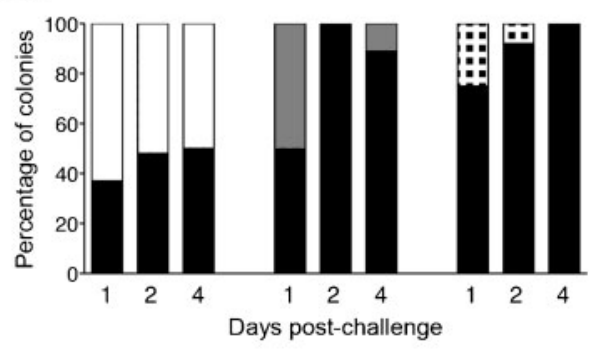

(b)

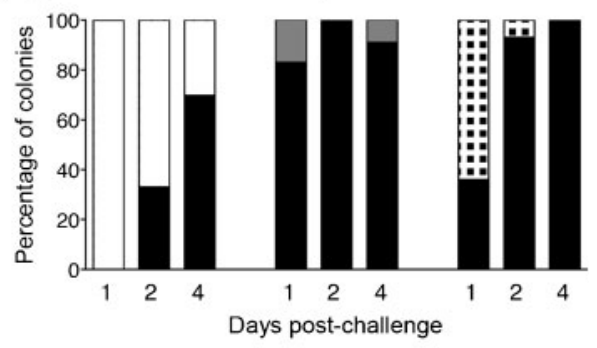

(c)

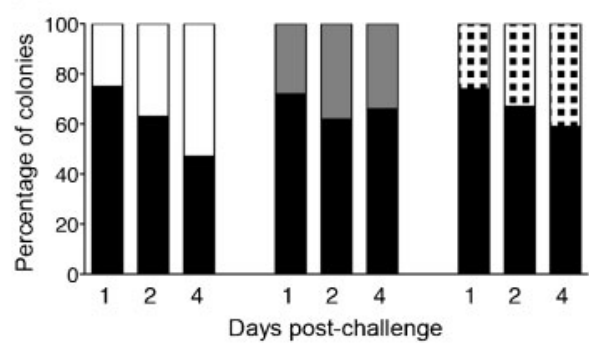

Fig. 4. Co-challenge trial: colonization levels after enrichment. Colonization of the liver and spleen of challenged birds was often quite low; therefore, portions of the liver and spleen were enriched in selenite broth to increase detection sensitivity. (a) Salmonella colonies found in the liver on days 1, 2 and 4 after challenge with equal amounts of: wild-type Sal18 attTn7::tet and wild-type Sal18 attTn7:: cat (left-hand bars), wild-type Sal18 attTn7:: tet and Sal18 attTn7:: cat $\Delta s p a S \Delta s s a U$ (centre bars), and wild-type Sal18 attTn $7:$ :tet and Sal18 $\Delta$ SPI-1 $\Delta$ SPI-2::cat (right-hand bars). Panels (b) and (c) have the same data arrangement, but depict the colonization profiles of the spleen and caecum, respectively. Black bars, Sal18 attTn7::tet; white bars, Sal18 attTn $7:$ : cat; grey bars, Sal18 attTn $7::$ cat $\Delta s p a S \Delta s s a U$; dotted bars, Sal18 attTn $7:$ : tet and Sal18 $\Delta$ SPI-1 $\Delta$ SPI-2 : : cat.

no significant differences between the strains were observed on days 3 and 4 post-challenge.

\section{DISCUSSION}

Prior to this study no knockout mutants of the complete SPI-2 region in S. Enteritidis had been reported. However, very recent work by Rychlik and co-workers, published after completion of our animal trials, describes the (a) Liver

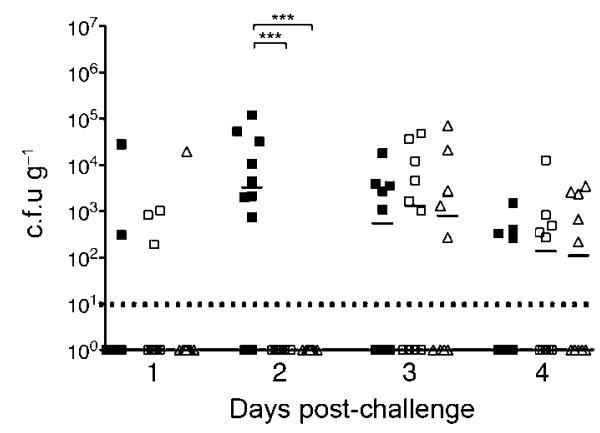

(b) Spleen

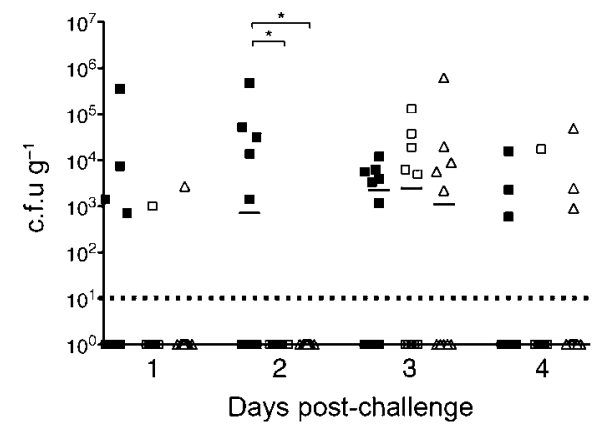

(c)

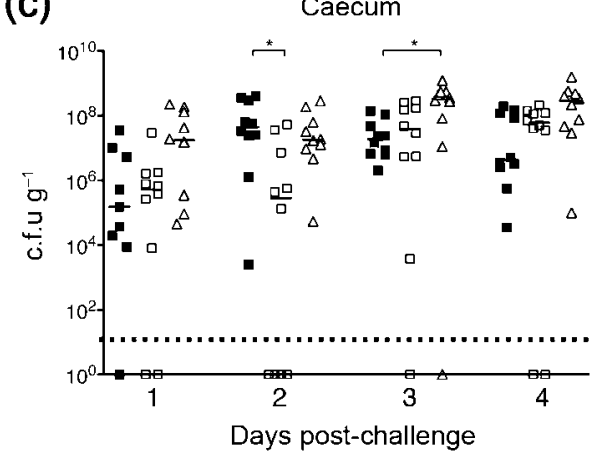

Fig. 5. Single-challenge trial: recovery of Salmonella from liver, spleen and caecum. (a) Calculated c.f.u. $\mathrm{g}^{-1}$ in the livers of birds challenged with wild-type Sal18 attTn7::tet (ם), Sal18 $\Delta$ SPI$2:$ : cat $(\square)$ and Sal18 $\Delta$ SPI- $1 \Delta$ SPI- $2:$ :cat $(\triangle)$ on days $1,2,3$ and 4 after challenge. Panels (b) and (c) show the c.f.u. $\mathrm{g}^{-1}$ in the spleen and caecum of birds. We were unable to detect $S$. Enteritidis counts below $10^{1}$ c.f.u. $\mathrm{g}^{-1}$ by direct plating with our sampling method. This is shown by the dotted lines in the figure. Birds negative for countable c.f.u. after direct plating on Brilliant Green agar were assigned a value of 1 for graphical purposes. Asterisks indicate degree of statistical significance: ${ }^{*}, P<0.05$; ${ }^{\star *}, P<0.01 ;{ }^{* \star}, P<0.001$.

complete knockout of all the SPI regions (together and separately), and their effect on colonization of 1-day-old chicks (Rychlik et al., 2009). Also, at least partial deletions of SPI-2 have been produced in S. Typhimurium, as well as full deletions of SPI-1 (Dieye et al., 2009; Hansen-Wester et al., 2004; Lawley et al., 2006). Using the $\lambda$ Red system, 

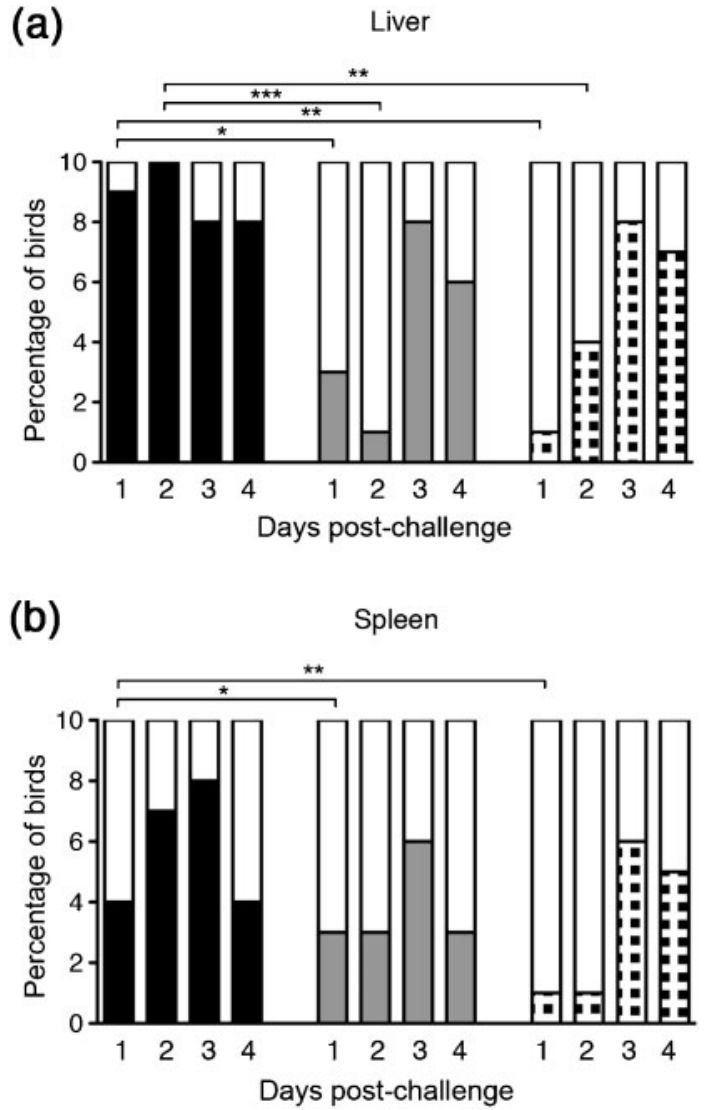

Fig. 6. Single-challenge trial: colonization levels after enrichment. (a) Number of birds positive or negative for the challenge strain in the liver on days 1, 2, 3 and 4 post-challenge. (b) Number of birds positive or negative for the challenge strain in the spleen. Asterisks indicate degree of statistical significance: ${ }^{*}, P<0.05$; ${ }^{\star \star}, P<0.01$; ${ }^{\star \star *}, P<0.001$. Black bars, Sal18 attTn7:: tet; grey bars, Sal18 $\Delta$ SPI-2 : : cat; dotted bars, Sal18 $\Delta$ SPI-1 $\Delta$ SPI-2 : : cat; white bars, negative for Salmonella.

developed by Datsenko \& Wanner (2000), derivative strains of Sal18 lacking either one or both of the entire SPI-1 and SPI-2 regions (Sal18 $\Delta$ SPI-2::cat and Sal18 $\Delta$ SPI-1 $\Delta$ SPI-2::cat) were constructed.

An earlier study, testing colonization levels of 1-week-old chickens challenged with $S$. Enteritidis strain 1009 on days $7,14,28,35$ and 42 post-challenge, showed maximal colonization levels of the spleen and caecum on day 7 (Sadeyen et al., 2004). Those authors observed similar results when working with 30-week-old chickens challenged with the same bacterial strain (Sadeyen et al., 2006). Another study testing layer hens challenged with $S$. Enteritidis Y-24 (phage type 4) showed that the highest colonization in the caecum was achieved on days 3 and 4 post-challenge (Piao et al., 2007). As the maximum colonization happened by 1 week, we believed that it was important to monitor the window between days 1 and 7 post-challenge. In preliminary trials done by our group (data not shown), we found that by days 3 and 4 postchallenge, strain Sal18 was beginning to clear from the liver, spleen and caecum of the chickens. Therefore, days 1 , 2,3 and 4 post-challenge were chosen for further trials. The challenge dose used in these studies was quite high $\left(10^{10}\right.$ c.f.u.) compared with those of similar studies, which have tended to range from $10^{4}$ to $10^{8}$ c.f.u. (Berndt et al., 2007; Bohez et al., 2006; Sadeyen et al., 2004, 2006). Preliminary experiments done by our group (data not shown) involving the challenge of 21-day-old broiler chickens and the challenge of 1-day-old SPF chickens with $10^{6}, 10^{8}$ and $10^{10}$ c.f.u. of wild-type $S$. Enteritidis strain Sal18 indicated that oral challenge with $10^{10}$ c.f.u. of the strain yielded the highest colonization levels of liver and spleen, while caecal colonization levels remained the same at all doses.

The results of the co-challenge trial showed that when 35day-old SPF chickens were challenged with both a wildtype strain and a strain impaired in the SPI- 1 and SPI-2 T3SSs, the wild-type strain began to outcompete the mutant strain in the liver and spleen by day 2 postchallenge (Fig. 3b, c). However, there was no detectable difference in the level of wild-type versus mutant strain in the caecal contents (Fig. 3b, c). A similar experimental design has been used by Dieye et al. (2009) in a recent study comparing colonization levels of wild-type $S$. Typhimurium UK-1, a $\Delta$ SPI-1 strain lacking the entire SPI-1 region, a $\Delta$ SPI-2 strain lacking a portion of the SPI-2 region encoding structural genes, and a combination $\Delta$ SPI$1 \Delta$ SPI-2 strain. In that study, 1-week-old SPF chickens were co-challenged with different combinations of the above strains, and chickens were euthanized at days 3 , 7 and 14 post-infection to test colonization levels in the spleen and caecum. Similar to our findings, that group recovered greater numbers of the wild-type $S$. Typhimurium strain than the $\Delta \mathrm{SPI}-2$ and $\Delta \mathrm{SPI}-1 \Delta \mathrm{SPI}-2$ strains from the spleen. They also observed that colonization levels of the wild-type versus the mutant strains in the caecum were not different, again supporting our results (Dieye et al., 2009). The spaS and ssaU genes encode structural proteins of SPI-1 and SPI-2 T3SSs, respectively. The spaS gene is last in the inv-spa operon of SPI-1. The ssaU gene is likewise positioned at the end of the $s s a K-U$ operon of SPI-2. Deletion of the spaS and/or ssaU genes should stop secretion of both SPI-1 and SPI-2 secreted proteins, but should not stop transcription of other genes in the SPI-1 or SPI-2 regions, or elsewhere in the chromosome (Hensel et al., 1997; Hueck, 1998; Jones et al., 2001, 2007; Rhen \& Dorman, 2005). Our study comparing the $\Delta$ spaS $\Delta s s a U$ mutant strain with the $\Delta$ SPI$1 \Delta$ SPI-2 strain in vivo showed that the strain with only the two genes deleted behaved in a similar way to the strain lacking the entirety of both islands when given as a cochallenge with a wild-type strain.

The results of the single-challenge trial indicate that strains lacking either the SPI-2 or both the SPI-1 and SPI-2 regions are impaired in their ability to infect the liver and spleen of SPF chickens. On day 1 post-challenge, both 
the wild-type and the mutant strains began to spread systemically to organs (liver and spleen), and by day 3 the wild-type strain was beginning to clear. While the wildtype strain reached peak colonization of the liver and spleen by day 2 , the mutant strains did not reach their peak until day 3 . By day 4 , both the wild-type and the mutant strains were clearing from the birds (Figs 5 and 6). Bohez and colleagues have observed that the maximum presence of Salmonella in the liver and spleen of SPF birds challenged with $10^{8}$ c.f.u. S. Enteritidis on the day of hatching occurs on day 2, supporting our observations (Bohez et al., 2006). A study by Jones et al. (2007) found that when SPF chickens were challenged with $S$. Typhimurium F98 at 1 week of age, the chickens had a peak colonization of the liver and caecum at 3 days postchallenge. When challenged with the same strain impaired in the SPI-1 region $(\Delta s p a S)$, peak colonization was again observed 3 days post-challenge, but seemed to have cleared faster in the liver and spleen. In chickens receiving a strain impaired in the SPI-2 region $(\Delta s s a U)$, systemic infection was not observed, and clearance of the strain from the caecum was evident by day 7 (Jones et al., 2007). This is partly in accordance with our results presented here. Our observations indicate that mutant strains are cleared faster from the liver and spleen than the wild-type strain. However, while Jones et al. (2007) observed a difference in caecal colonization between strains, we did not make a similar observation in either of our trials. This may be attributed to a difference in strain (those studies used an isolate of $S$. Typhimurium, while ours used $S$. Enteritidis) or differences in challenge dose.

As mentioned above, we have observed that the SPI-2 T3SS is not important for caecal colonization, and while the SPI2 T3SS does appear to be important for efficient systemic infection, it is obviously not the only factor involved. These observations are in line with the results of another group who found that neither the SPI-1 nor the SPI-2 T3SS of an $S$. Typhimurium strain was essential to the invasion of $\mathrm{M}$ cells in vitro (Martinez-Argudo \& Jepson, 2008). Their studies used an in vitro model involving the co-culture of Caco-2 cells with Raji B cells (which causes the Caco-2 cells to exhibit traits of $\mathrm{M}$ cells) in order to better mimic the intestinal environment that Salmonella encounters in the human host. Another group using an in vitro model found that while the SPI-2 T3SS was expressed at all times within the SCVs of mouse macrophage cells, the expression levels in the SCVs of human epithelial cells were reduced compared with those in the macrophages (Hautefort et al., 2008). This further supports our conclusion that SPI-2 is important for systemic infection in chickens, but not essential for caecal colonization.

The recent study by Rychlik et al. (2009) used S. Enteritidis strain 147 SPI mutants lacking one of the islands (SPI-1 to -5), lacking both SPI-1 and SPI-2, lacking all islands (SPI-1 to -5 ), retaining one of the islands only (SPI-1 to -5 ), or retaining SPI-1 and SPI-2 only. They tested the ability of these mutants (compared with the wild-type strain) to colonize the liver and spleen of 1-day-old chicks. They found that after oral challenge with $5 \times 10^{7}$ c.f.u., the strains lacking SPI-1 or SPI-2, and the strains containing SPI-1 or SPI-2 only, maintained a medium level of virulence, and were found in the liver and spleen of challenged chickens (although in lower numbers than the wild-type strain) at both 5 and 7 days after challenge. However, the strain lacking all of the pathogenicity islands (SPI-1 to -5), and those containing only SPI-3, SPI-4 or SPI-5, were avirulent, and were isolated from the liver and spleen only in very small amounts. Strains lacking SPI-3, SPI-4 or SPI-5 were isolated from the liver and spleen at the same levels as the wild-type strain. They also found that the strains lacking both SPI-1 and SPI-2 were not isolated from the liver and spleen as much as the wild-type strain, or the strain containing the SPI-1 and SPI-2 regions only. Their study indicates the importance of both SPI-1 and SPI-2 in colonization of the liver and spleen, but also shows that although at much lower levels than the wild-type, mutants lacking one or both of SPI-1 and SPI- 2 can still be isolated from the liver and spleen, further confirming our results. They found no difference in caecal colonization by any of their strains, in accordance with our results (Rychlik et al., 2009).

A study by Morgan et al. (2004) involved screening a large number of $S$. Typhimurium mutants, including several single-gene knockouts in the SPI-1 and SPI-2 regions. They found that their SPI-1 and SPI-2 mutants were not effective colonizers of bovine ileum between 3 and 5 days postinfection. At first glance, their results seem to contradict our findings. However, they found that the majority of the SPI-1 and SPI-2 mutants tested were able to successfully colonize the caecum of 14-day-old chickens. They also tested a number of other mutants, including single-gene knockouts of certain surface structures, and found that while many of these mutants were equivalent to the wildtype strain in their ability to colonize cattle, they were attenuated in chickens. In addition, they observed that mutations of the SPI-4 region caused attenuation in cattle, but not in chickens, further demonstrating that Salmonella must use different approaches for colonization or infection of different hosts. This is not surprising, as $S$. Typhimurium causes systemic disease in cattle, while it does not cause disease in chickens older than 1 week of age (Morgan et al., 2004). Although S. Typhimurium and S. Enteritidis are considered to be distinct serovars, they are very close genetically, and can cause similar disease in humans. The fact that Morgan et al. (2004) found no difference in colonization of chicken caeca between their wild-type $S$. Typhimurium strain and their SPI-1 and SPI-2 mutants matches our results with $S$. Enteritidis in both 7and 35-day-old chickens, and validates our finding that the SPI-2 T3SS is not essential for colonization of chickens. In addition, their observation that deletions of certain fimbrial and other structural genes cause attenuation of the strain in chickens may be the answer to how these strains colonize chickens without the use of the SPI-1 and 
SPI-2 T3SSs. Further work must be done in this area to identify the exact method used by $S$. Typhimurium and $S$. Enteritidis to colonize the chicken caecum, as well as spread systemically in these animals.

In summary, the results of our studies indicate that in a cochallenge situation, in 35-day-old chickens, wild-type strains of $S$. Enteritidis are more competitive than strains impaired in the SPI-1 and SPI-2 regions. Also, we showed that in a single-challenge model, in 1-week-old chicks, mutants lacking either the SPI-2 region or both the SPI-1 and the SPI-2 region are initially impaired in their ability to invade the liver and spleen compared with the wild-type strain, although they remain present in the caecum at levels similar to those of the wild-type strain.

\section{ACKNOWLEDGEMENTS}

Thanks are due to: Mr Andrew Shih for some of the initial steps in the cloning; Dr Aaron P. White and Dr Michael G. Surette (University of Calgary, Calgary, AB, Canada) for the plasmid pHSG415, as well as the $\lambda$ Red plasmids; the Animal Care Unit, Vaccine and Infectious Disease Organization, University of Saskatchewan, for the raising of antibodies and excellent technical support in the animal trials; $\mathrm{Dr}$ Hugh Townsend for the direction in statistical analysis; and Dr Brenda Allan for helpful discussions. This project is supported by the Natural Sciences and Engineering Research Council of Canada (NSERC) and Bioniche Life Sciences, Inc. A. A. P. holds an NSERC Senior Industrial Research Chair in food and water safety vaccines; W. K. holds an NSERC Associate Industrial Research Chair in food and water safety vaccines. W.K. received a New Investigator Establishment Grant from the Saskatchewan Health Research Foundation (SHRF).

\section{REFERENCES}

Babu, U. S., Gaines, D. W., Lillehoj, H. \& Raybourne, R. B. (2006). Differential reactive oxygen and nitrogen production and clearance of Salmonella serovars by chicken and mouse macrophages. Dev Comp Immunol 30, 942-953.

Berndt, A., Wilhelm, A., Jugert, C., Pieper, J., Sachse, K. \& Methner, U. (2007). Chicken cecum immune response to Salmonella enterica serovars of different levels of invasiveness. Infect Immun 75, 59936007.

Bohez, L., Ducatelle, R., Pasmans, F., Botteldoorn, N., Haesebrouck, F. \& Van Immerseel, F. (2006). Salmonella enterica serovar Enteritidis colonization of the chicken caecum requires the HilA regulatory protein. Vet Microbiol 116, 202-210.

Brawn, L. C., Hayward, R. D. \& Koronakis, V. (2007). Salmonella SPI1 effector SipA persists after entry and cooperates with a SPI2 effector to regulate phagosome maturation and intracellular replication. Cell Host Microbe 1, 63-75.

Brown, N. F., Vallance, B. A., Coombes, B. K., Valdez, Y., Coburn, B. A. \& Finlay, B. B. (2005). Salmonella pathogenicity island 2 is expressed prior to penetrating the intestine. PLoS Pathog 1, e32.

Callaway, T. R., Edrington, T. S., Anderson, R. C., Byrd, J. A. \& Nisbet, D. J. (2008). Gastrointestinal microbial ecology and the safety of our food supply as related to Salmonella. J Anim Sci 86, E163E172.

Catarame, T. M. G., O'Hanlon, K. A., McDowell, D. A., Blair, I. S. \& Duffy, G. (2005). Comparison of a real-time polymerase chain reaction assay with a culture method for the detection of Salmonella in retail meat samples. J Food Saf 26, 1-15.

Chakravortty, D., Rohde, M., Jager, L., Deiwick, J. \& Hensel, M. (2005). Formation of a novel surface structure encoded by Salmonella pathogenicity island 2. EMBO J 24, 2043-2052.

Clavijo, R. I., Loui, C., Andersen, G. L., Riley, L. W. \& Lu, S. (2006). Identification of genes associated with survival of Salmonella enterica serovar Enteritidis in chicken egg albumen. Appl Environ Microbiol 72, 1055-1064.

Coombes, B. K. \& Finlay, B. B. (2005). Insertion of the bacterial type III translocon: not your average needle stick. Trends Microbiol 13, 92 95.

Coombes, B. K., Brown, N. F., Valdez, Y., Brumell, J. H. \& Finlay, B. B. (2004). Expression and secretion of Salmonella pathogenicity island-2 virulence genes in response to acidification exhibit differential requirements of a functional type III secretion apparatus and SsaL. J Biol Chem 279, 49804-49815.

Coombes, B. K., Coburn, B. A., Potter, A. A., Gomis, S., Mirakhur, K., Li, Y. \& Finlay, B. B. (2005). Analysis of the contribution of Salmonella pathogenicity islands 1 and 2 to enteric disease progression using a novel bovine ileal loop model and a murine model of infectious enterocolitis. Infect Immun 73, 7161-7169.

Craig, N. L. (1991). Tn7: a target site-specific transposon. Mol Microbiol 5, 2569-2573.

Datsenko, K. A. \& Wanner, B. L. (2000). One-step inactivation of chromosomal genes in Escherichia coli K-12 using PCR products. Proc Natl Acad Sci U S A 97, 6640-6645.

Desin, T. S., Lam, P. K., Koch, B., Mickael, C., Berberov, E., Wisner, A. L., Townsend, H. G., Potter, A. A. \& Köster, W. (2009). Salmonella enterica serovar Enteritidis pathogenicity island 1 is not essential for but facilitates rapid systemic spread in chickens. Infect Immun 77, 2866-2875.

Dieye, Y., Ameiss, K., Mellata, M. \& Curtiss, R., III (2009). The Salmonella pathogenicity island (SPI) 1 contributes more than SPI2 to the colonization of the chicken by Salmonella enterica serovar Typhimurium. BMC Microbiol 9, 3.

el Yaagoubi, A., Kohiyama, M. \& Richarme, G. (1994). Localization of DnaK (chaperone 70) from Escherichia coli in an osmotic-shocksensitive compartment of the cytoplasm. J Bacteriol 176, 7074-7078.

Foley, S. L., Lynne, A. M. \& Nayak, R. (2008). Salmonella challenges: prevalence in swine and poultry and potential pathogenicity of such isolates. J Anim Sci 86, E149-E162.

Fortune, D. R., Suyemoto, M. \& Altier, C. (2006). Identification of $\mathrm{CsrC}$ and characterization of its role in epithelial cell invasion in Salmonella enterica serovar Typhimurium. Infect Immun 74, 331-339.

Galán, J. E. (2001). Salmonella interactions with host cells: type III secretion at work. Annu Rev Cell Dev Biol 17, 53-86.

Ghosh, P. (2004). Process of protein transport by the type III secretion system. Microbiol Mol Biol Rev 68, 771-795.

Giacomodonato, M. N., Uzzau, S., Bacciu, D., Caccuri, R., Sarnacki, S. H., Rubino, S. \& Cerquetti, M. C. (2007). SipA, SopA, SopB, SopD and SopE2 effector proteins of Salmonella enterica serovar Typhimurium are synthesized at late stages of infection in mice. Microbiology 153, 1221-1228.

Guiney, D. G. (2005). The role of host cell death in Salmonella infections. Curr Top Microbiol Immunol 289, 131-150.

Hansen-Wester, I., Chakravortty, D. \& Hensel, M. (2004). Functional transfer of Salmonella pathogenicity island 2 to Salmonella bongori and Escherichia coli. Infect Immun 72, 2879-2888.

Hautefort, I., Thompson, A., Eriksson-Ygberg, S., Parker, M. L., Lucchini, S., Danino, V., Bongaerts, R. J., Ahmad, N., Rhen, M. \& 
other authors (2008). During infection of epithelial cells Salmonella enterica serovar Typhimurium undergoes a time-dependent transcriptional adaptation that results in simultaneous expression of three type 3 secretion systems. Cell Microbiol 10, 958-984.

Hensel, M., Shea, J. E., Raupach, B., Monack, D., Falkow, S., Gleeson, C., Kubo, T. \& Holden, D. W. (1997). Functional analysis of ssaJ and the ssaK/U operon, 13 genes encoding components of the type III secretion apparatus of Salmonella pathogenicity island 2. Mol Microbiol 24, 155-167.

Hueck, C. J. (1998). Type III protein secretion systems in bacterial pathogens of animals and plants. Microbiol Mol Biol Rev 62, 379-433.

Jones, M. A., Wigley, P., Page, K. L., Hulme, S. D. \& Barrow, P. A. (2001). Salmonella enterica serovar Gallinarum requires the Salmonella pathogenicity island 2 type III secretion system but not the Salmonella pathogenicity island 1 type III secretion system for virulence in chickens. Infect Immun 69, 5471-5476.

Jones, M. A., Hulme, S. D., Barrow, P. A. \& Wigley, P. (2007). The Salmonella pathogenicity island 1 and Salmonella pathogenicity island 2 type III secretion systems play a major role in pathogenesis of systemic disease and gastrointestinal tract colonization of Salmonella enterica serovar Typhimurium in the chicken. Avian Pathol 36, 199203.

Kuhle, V. \& Hensel, M. (2004). Cellular microbiology of intracellular Salmonella enterica: functions of the type III secretion system encoded by Salmonella pathogenicity island 2. Cell Mol Life Sci 61, 28122826.

Lawley, T. D., Chan, K., Thompson, L. J., Kim, C. C., Govoni, G. R. \& Monack, D. M. (2006). Genome-wide screen for Salmonella genes required for long-term systemic infection of the mouse. PLoS Pathog 2, e11.

Lee, Y. J., Mo, I. P. \& Kang, M. S. (2005). Safety and efficacy of Salmonella gallinarum $9 \mathrm{R}$ vaccine in young laying chickens. Avian Pathol 34, 362-366.

Martinez-Argudo, I. \& Jepson, M. A. (2008). Salmonella translocates across an in vitro $M$ cell model independently of SPI-1 and SPI-2. Microbiology 154, 3887-3894.

McGhie, E. J., Brawn, L. C., Hume, P. J., Humphreys, D. \& Koronakis, V. (2009). Salmonella takes control: effector-driven manipulation of the host. Curr Opin Microbiol 12, 117-124.

Meenakshi, M., Bakshi, C. S., Butchaiah, G., Bansal, M. P., Siddiqui, M. Z. \& Singh, V. P. (1999). Adjuvanted outer membrane protein vaccine protects poultry against infection with Salmonella enteritidis. Vet Res Commun 23, 81-90.

Morgan, E., Campbell, J. D., Rowe, S. C., Bispham, J., Stevens, M. P., Bowen, A. J., Barrow, P. A., Maskell, D. J. \& Wallis, T. S. (2004). Identification of host-specific colonization factors of Salmonella enterica serovar Typhimurium. Mol Microbiol 54, 994-1010.

Murphy, K. C. \& Campellone, K. G. (2003). Lambda Red-mediated recombinogenic engineering of enterohemorrhagic and enteropathogenic E. coli. BMC Mol Biol 4, 11.

Olekhnovich, I. N. \& Kadner, R. J. (2006). Crucial roles of both flanking sequences in silencing of the hilA promoter in Salmonella enterica. J Mol Biol 357, 373-386.

Piao, Z., Toyota-Hanatani, Y., Ohta, H., Sasai, K., Tani, H. \& Baba, E. (2007). Effects of Salmonella enterica subsp. enterica serovar Enteritidis vaccination in layer hens subjected to $S$. Enteritidis challenge and various feed withdrawal regimens. Vet Microbiol 125, $111-119$.

Rana, N. \& Kulshreshtha, R. C. (2006). Cell-mediated and humoral immune responses to a virulent plasmid-cured mutant strain of Salmonella enterica serotype Gallinarum in broiler chickens. Vet Microbiol 115, 156-162.
Rhen, M. \& Dorman, C. J. (2005). Hierarchical gene regulators adapt Salmonella enterica to its host milieus. Int J Med Microbiol 294, 487502.

Rychlik, I., Karasova, D., Sebkova, A., Volf, J., Sisak, F., Havlickova, H., Kummer, V., Imre, A., Szmolka, A. \& other authors (2009). Virulence potential of five major pathogenicity islands (SPI-1 to SPI-5) of Salmonella enterica serovar Enteritidis for chickens. BMC Microbiol 9, 268.

Sadeyen, J. R., Trotereau, J., Velge, P., Marly, J., Beaumont, C., Barrow, P. A., Bumstead, N. \& Lalmanach, A. C. (2004). Salmonella carrier state in chicken: comparison of expression of immune response genes between susceptible and resistant animals. Microbes Infect 6, 1278-1286.

Sadeyen, J. R., Trotereau, J., Protais, J., Beaumont, C., Sellier, N., Salvat, G., Velge, P. \& Lalmanach, A. C. (2006). Salmonella carrierstate in hens: study of host resistance by a gene expression approach. Microbes Infect 8, 1308-1314.

Shah, D. H., Lee, M. J., Park, J. H., Lee, J. H., Eo, S. K., Kwon, J. T. \& Chae, J. S. (2005). Identification of Salmonella gallinarum virulence genes in a chicken infection model using PCR-based signature-tagged mutagenesis. Microbiology 151, 3957-3968.

Schlumberger, M. C. \& Hardt, W. D. (2006). Salmonella type III secretion effectors: pulling the host cell's strings. Curr Opin Microbiol 9, 46-54.

Thompson, A., Rowley, G., Alston, M., Danino, V. \& Hinton, J. C. (2006). Salmonella transcriptomics: relating regulons, stimulons and regulatory networks to the process of infection. Curr Opin Microbiol 9, 109-116.

Thomson, N. R., Clayton, D. J., Windhorst, D., Vernikos, G., Davidson, S., Churcher, C., Quail, M. A., Stevens, M., Jones, M. A. \& other authors (2008). Comparative genome analysis of Salmonella Enteritidis PT4 and Salmonella Gallinarum 287/91 provides insights into evolutionary and host adaptation pathways. Genome Res 18, 1624-1637.

Tischer, B. K., von Einem, J., Kaufer, B. \& Osterrieder, N. (2006). Two-step red-mediated recombination for versatile high-efficiency markerless DNA manipulation in Escherichia coli. Biotechniques 40, 191-197.

Turner, A. K., Lovell, M. A., Hulme, S. D., Zhang-Barber, L. \& Barrow, P. A. (1998). Identification of Salmonella typhimurium genes required for colonization of the chicken alimentary tract and for virulence in newly hatched chicks. Infect Immun 66, 2099-2106.

Vieira, A. \& other authors (2009). WHO Global Foodborne Infections Network Country Databank - a resource to link human and nonhuman sources of Salmonella. XII International Society for Veterinary Epidemiology and Economics Conference. http://www.who.int/gfn/ activities/CDB_poster_Sept09.pdf

Waterman, S. R. \& Holden, D. W. (2003). Functions and effectors of the Salmonella pathogenicity island 2 type III secretion system. Cell Microbiol 5, 501-511.

White, A. P., Allen-Vercoe, E., Jones, B. W., DeVinney, R., Kay, W. W. \& Surette, M. G. (2007). An efficient system for markerless gene replacement applicable in a wide variety of enterobacterial species. Can J Microbiol 53, 56-62.

Wigley, P., Jones, M. A. \& Barrow, P. A. (2002). Salmonella enterica serovar Pullorum requires the Salmonella pathogenicity island 2 type III secretion system for virulence and carriage in the chicken. Avian Pathol 31, 501-506.

Winstanley, C. \& Hart, C. A. (2001). Type III secretion systems and pathogenicity islands. J Med Microbiol 50, 116-126.

Edited by: V. J. Cid 